\title{
On the Magnetogravitational Instability of a Ferromagnetic Dust Cloud in the Presence of Nonuniform Rotation
}

\author{
Joginder S. Dhiman and Rekha Dadwal \\ Department of Mathematics, Himachal Pradesh University, Summer Hill, Shimla 171005, India \\ Correspondence should be addressed to Joginder S. Dhiman, jsdhiman66@gmail.com \\ Received 10 April 2012; Accepted 6 June 2012 \\ Academic Editors: S. Bogovalov, C. W. Engelbracht, and R. Hawkes
}

Copyright ( $) 2012$ J. S. Dhiman and R. Dadwal. This is an open access article distributed under the Creative Commons Attribution License, which permits unrestricted use, distribution, and reproduction in any medium, provided the original work is properly cited.

The effect of a nonuniform magnetic field on the gravitational instability of a nonuniformly rotating infinitely extending axisymmetric cylinder in a homogenous ferromagnetic medium has been studied. The propagation of the wave is allowed along radial direction. A general dispersion relation, using the normal mode analysis method on the perturbation equations of the problem, is obtained. It is found that Bel and Schatzman criterion determines the gravitational instability of this general problem. Thus, it appears that the effect of non-uniform magnetic field on the gravitational instability as discussed by (Dhiman and Dadwal, 2010 ) is marginalized by the magnetic polarizability of ferrofluid.

\section{Introduction}

The gravitational instability (GI) or the Jeans instability causes the collapse of interstellar gas clouds and subsequent star formation. It occurs when the internal gas pressure is not strong enough to prevent gravitational collapse of a region filled with matter. GI plays a dominant role in determining the star formation properties of galaxies. The problem of instability of self-gravitating large gas clouds was first considered by Jeans [1] and underlined the importance of self-gravitating instabilities in astrophysics because of their crucial role in understanding collapse, formation, and evolution of interstellar molecular clouds, star formation, galactic structure and its evolution, and so forth. For a latest and broader review on the subject of gravitational instability, one may refer to [1-8] and references therein.

Theoretical studies have shown that the magnetic field plays a vital role in self-gravitating star-forming regions and in the evolution of interstellar clouds into self-gravitating star forming regions. The problem of magnetogravitational instability of interstellar rotating medium is of considerable importance in connection with the protostar and star formation in magnetic dust clouds. In astrophysics, the problems that are considered generally assume magnetic field/rotation to be uniform: however, this idealization of the uniform character in theoretical investigations is valid only for laboratory purposes (cf. Larson [8] and references within), because in the interstellar interior and atmosphere, the magnetic field/rotation may be variable and may together alter the nature of the instability. Limited efforts have been put to investigate the GI by considering the magnetic field or rotation to be variable. Bel and Schatzman [9] studied the effect of nonuniform rotation on the onset of gravitational instability and obtained a modified expression for the Jeans criterion, which is now known as the Bel and Schatzman criterion. Recently, Dhiman and Dadwal $[10,11]$ also studied the simultaneous effect of nonuniform magnetic field and rotation on the gravitational instability of gaseous medium and obtained some general qualitative results.

In astronomy, the interstellar medium (ISM) is the matter that exists in the space between the star systems in a galaxy. This matter includes gas in ionic, atomic, and molecular form, dust, and cosmic rays. It fills interstellar space and blends smoothly into the surrounding intergalactic space. The dust, which is composed of small solid particles by segregation in the interstellar clouds, is a very important component of the ISM. In the recent past, there have been dramatic changes in the conception of the interstellar medium, and recent observational and numerical works have suggested that interstellar medium (ISM) plays 
an important role in the star formation. Spitzer [12] has shown that solid particles in space including dust grains are eclectically charged, relative to surrounding plasma. Further, the efficiency and timescale of stellar birth in Galactic molecular clouds strongly depend on the properties of interstellar medium (ISM).

Ferrofluids are colloidal liquids made of nanoscale ferromagnetic, or ferrimagnetic, particles suspended in a carrier fluid (usually an organic solvent or water). A ferrofluid flow in the presence of an applied magnetic field is accompanied by an intertwinement of hydrodynamic and magnetic interactions (Rosenwieg [13]). A ferrofluid is stable and does not solidify even in the presence of a high magnetic field and is commonly characterized by its strong tendency to magnetize in the direction of the magnetic field. Jones and Spitzer [14] provided a model for the existence of gas-dust interstellar mediums with a highly pronounced property of magnetic polarizability. This can be assumed due to a super paramagnetic dispersion of the fine ferromagnetic grains suspended in a gaseous cloud of molecular hydrogen. Mamun and Shukla [15] observed the usual Jeans instability in a self-gravitating dark interstellar molecular cloud containing ferromagnetic dust grains and baryonic gas clouds and supported the existence of ferromagnetic dust particles in a magnetically supported dark interstellar self-gravitating interstellar molecular cloud.

The present study is primarily motivated by the investigations of Mamun and Shukla [15] regarding the instability of a self-gravitating dark interstellar molecular cloud containing ferromagnetic dust grains. Our aim here is to investigate the gravitational instability of an infinite axisymmetric cylinder of a homogenous nonuniformly rotating medium containing the ferromagnetic dust particles in the presence of nonuniform magnetic field. The mathematical analysis followed in this paper is precisely the same as that of [9, 10]. Since the system of linearized perturbation equation governing the problem contains variable coefficients, the sufficient condition for GI is derived using the local instability approach as adopted in [9].

\section{Mathematical Model and Basic Equations}

Consider an infinite homogeneous, interstellar selfgravitating molecular cloud containing ferromagnetic dust particles. The system is permeated with the simultaneous action of a nonuniform rotation and a nonuniform magnetic field. In a rectangular coordinate system, let $\vec{u}=\left(u_{x}, u_{y}, u_{z}\right)$ be the velocity, $\vec{M}=\left(M_{x}, M_{y}, M_{z}\right)$ be the ferrofluid magnetization under the magnetic field $\vec{H}=\left(H_{x}, H_{y}, H_{z}\right)$. For the treatment of this physical configuration for gravitational instability, the conventional set of ferrohydrodynamic equations are given by $(c f .[16,17])$

$$
\begin{gathered}
\frac{d \vec{u}}{d t}=-\operatorname{grad} p+\rho \operatorname{grad} \phi+\mu_{0}(\vec{M} \cdot \nabla) \vec{H} \\
\frac{\partial \rho}{\partial t}+(\vec{u} \cdot \operatorname{grad}) \rho+\rho(\nabla \cdot \vec{u})=0 \\
\nabla^{2} \phi=-4 \pi G\left(\rho-\rho_{0}\right) .
\end{gathered}
$$

The ferrofluid magnetization $\vec{M}$ satisfies Shliomis' [16, 17] equation of magnetization, which is given by

$$
\frac{d \vec{M}}{d t}=\frac{1}{2}(\nabla \times \vec{u}) \times \vec{M}-\alpha\left(\vec{M}-\vec{M}_{0}\right)-\beta \vec{M} \times(\vec{M} \times \vec{H}) .
$$

In the above equations $p, \phi, \mu_{0}, \rho$, and $G$, respectively, denote the pressure, gravitational potential, the inverse Brownian time constant for particle diffusion in the ferrofluid, the magnetic permeability, density, the gravitational constant, $\alpha=3 \eta V / k_{b} T$ is the Brownian time of rotational particle diffusion and $\beta=1 / 6 \eta \varphi$, where $\eta$ is the dynamic viscosity of the carrier fluid and $\varphi=n V$ is the volume fraction of magnetic grains in the liquid. Here, $n$ is the number density and $V$ the volume of a single particle. Also, $\rho_{0}$ and $\vec{M}_{0}=\left(0,0, M_{0}\right)$ are the equilibrium values of density and magnetization and $M_{0}$ the equilibrium magnetization of ferrofluid is related to the equilibrium magnetic felid $H_{0}$ by

$$
M_{0}=n \bar{M}\left(\operatorname{coth} \psi-\frac{1}{\psi}\right) \frac{H_{0}}{\left\|H_{0}\right\|},
$$

where $\bar{M}$ is the magnetic moment of single ferromagnetic particle and the nondimensional quantity $\psi$ is given by $\psi=$ $\mu_{0}\left(\bar{M}\left\|H_{0}\right\| / k_{b} T_{b}\right)$, where $k_{b}$ is the Boltzman constant and $T_{b}$ is temperature of the ferrofluid. Further, the Poisson's equation (3) is considered so as to avoid the "Jeans Swindle" (cf. Speigel and Thiffeault [7]).

In Chu formulation of electrodynamics (see Penfield and Haus [18]), the magnetic field $\vec{H}$, magnetization $\vec{M}$, and magnetic induction $\vec{B}$ are related by

$$
\vec{B}=\mu_{0}(\vec{H}+\vec{M}) .
$$

We know that the magnetic field $\vec{H}$ satisfies the following Maxwell's equations:

$$
\begin{gathered}
\nabla \times \vec{H}=0, \\
\nabla \cdot \vec{B}=0 .
\end{gathered}
$$

Equation (6), using (8) yields

$$
\nabla \cdot \vec{H}=-\nabla \cdot \vec{M}
$$

In the present analysis, we shall consider an infinite axisymmetric cylinder of homogeneous, infinitely conducting self-gravitating ferromagnetic dust cloud under the simultaneous effect of a nonuniform rotation and nonuniform magnetic field, therefore, transforming the above fundamental equations in cylindrical coordinates $(r, \theta, z)$. Denoting $\vec{u}=\left(u_{r}, u_{\theta}, u_{z}\right)$ and $\vec{M}=\left(M_{r}, M_{\theta}, M_{z}\right)$ by the components of velocity $\vec{u}$ and ferrofluid magnetization $\vec{M}$ along the radial $r$, the transverse $\theta$, and the axial $z$ directions, respectively. The cylinder is assumed to be rotating about its axis ( $z$-axis) with nonuniform angular velocity $\omega$. The propagation of wave is taken along the radial direction " $r$ " 
of the cylinder, hence $\partial / \partial r$ is the only nonzero component of the gradient.

Following Dhiman and Dadwal [10], the basic equations (1)-(4), (7) and (9) under these assumptions take the following forms in cylindrical polar coordinates

$$
\begin{aligned}
& \rho\left(\frac{\partial u_{r}}{\partial t}+u_{r} \frac{\partial u_{r}}{\partial r}-\frac{u_{\theta}^{2}}{r}\right) \\
& =\rho \frac{\partial \phi}{\partial r}-\frac{\partial p}{\partial r}+\mu_{0}\left[M_{r} \frac{\partial H_{r}}{\partial r}-\frac{M_{\theta} H_{\theta}}{r}\right], \\
& \rho\left(\frac{\partial u_{\theta}}{\partial t}+u_{r} \frac{\partial}{\partial r} u_{\theta}+\frac{u_{r} u_{\theta}}{r}\right) \\
& =\frac{\mu_{0}}{2}\left[M_{r} \frac{\partial H_{\theta}}{\partial r}+H_{r} \frac{\partial M_{\theta}}{\partial r}+M_{\theta} \frac{\partial H_{r}}{\partial r}\right. \\
& \left.-H_{\theta} \frac{\partial M_{r}}{\partial r}+\frac{2 M_{\theta} H_{r}}{r}\right] \\
& \rho\left(\frac{\partial u_{z}}{\partial t}+u_{r} \frac{\partial u_{z}}{\partial r}\right) \\
& =\frac{\mu_{0}}{2}\left[M_{r} \frac{\partial H_{z}}{\partial r}+H_{r} \frac{\partial M_{z}}{\partial r}+M_{z} \frac{\partial H_{r}}{\partial r}-H_{z} \frac{\partial M_{r}}{\partial r}\right. \\
& \left.+\left(\frac{M_{z} H_{r}-H_{z} M_{r}}{r}\right)\right], \\
& \frac{\partial M_{r}}{\partial t}+u_{r} \frac{\partial M_{r}}{\partial r}+\frac{1}{2}\left(M_{\theta}\left(\frac{\partial u_{\theta}}{\partial r}+\frac{u_{\theta}}{r}\right)+M_{z} \frac{\partial u_{z}}{\partial r}\right) \\
& +\alpha\left(M_{r}-M_{0}\right)+\beta\left(M_{\theta}\left[M_{r} H_{\theta}-H_{r} M_{\theta}\right]\right. \\
& \left.-M_{z}\left[H_{r} M_{z}-M_{r} H_{z}\right]\right)=0, \\
& \frac{\partial M_{\theta}}{\partial t}+u_{r} \frac{\partial M_{\theta}}{\partial r}-\frac{1}{2}\left(M_{r}\left(\frac{\partial u_{\theta}}{\partial r}+\frac{u_{\theta}}{r}\right)\right) \\
& +\alpha\left(M_{\theta}-M_{0}\right)+\beta\left(M_{z}\left[M_{\theta} H_{z}-H_{\theta} M_{z}\right]\right. \\
& \left.-M_{r}\left[M_{r} H_{\theta}-H_{r} M_{\theta}\right]\right)=0, \\
& \frac{\partial M_{z}}{\partial t}+u_{r} \frac{\partial M_{z}}{\partial r}-\frac{1}{2}\left(M_{r} \frac{\partial u_{z}}{\partial r}\right)+\alpha\left(M_{z}-M_{0}\right) \\
& +\beta\left(M_{r}\left[H_{r} M_{z}-M_{r} H_{z}\right]\right. \\
& \left.-M_{\theta}\left[M_{\theta} H_{Z}-H_{\theta} M_{z}\right]\right)=0, \\
& \frac{\partial H_{z}}{\partial r}=0, \\
& \left(\frac{\partial H_{\theta}}{\partial r}+\frac{H_{\theta}}{r}\right)=0 \\
& \left(\frac{\partial H_{r}}{\partial r}+\frac{H_{r}}{r}\right)=-\left(\frac{\partial M_{r}}{\partial r}+\frac{M_{r}}{r}\right), \\
& \frac{\partial \rho}{\partial t}+(\vec{u} \cdot \operatorname{grad}) \rho+\rho(\nabla \cdot \vec{u})=0, \\
& \nabla^{2} \phi=-4 \pi G\left(\rho-\rho_{0}\right) .
\end{aligned}
$$

In these equations, the operators $\nabla$ and $\nabla^{2}$ now are respective operators in cylindrical coordinates.

\section{Equilibrium State and \\ Perturbation Equations}

Following the physical models of Mamun and Shukla [15] and Kumar et al. [19], the magnetization is taken along $r$ and $z$ directions.

The equilibrium state under discussion is clearly characterized as follows:

$$
\begin{gathered}
\vec{u}=(0, r \omega, 0) ; \quad \vec{H}=\left(0,0, H_{z}\right) ; \quad \vec{M}=\left(M_{r}, 0, M_{z}\right) \\
p=p_{0} ; \quad \phi=\phi_{0} ; \quad \rho=\rho_{0}
\end{gathered}
$$

Using (11) in (10) and using the fact that propagation of wave is along $r$ direction and thus $\partial / \partial r$ is the only nonzero component, we obtain the following basic solution:

$$
\begin{gathered}
\frac{\partial p_{0}}{\partial r}=\rho_{0}\left(\frac{\partial \phi_{0}}{\partial r}+r \omega^{2}\right) \\
M_{r} \frac{\partial H_{z}}{\partial r}-H_{z}\left(\frac{\partial M_{r}}{\partial r}+\frac{M_{r}}{r}\right)=0 \\
\alpha\left(M_{r}-M_{0}\right)+\beta M_{z} M_{r} H_{z}=0 \\
-\frac{1}{2} M_{r}\left(\frac{\partial u_{\theta}}{\partial r}+\frac{u_{\theta}}{r}\right)+\alpha M_{0}=0 \\
\alpha\left(M_{z}-M_{0}\right)-\beta M_{r}^{2} H_{z}=0 \\
\frac{\partial H_{z}}{\partial r}=0 \\
\left(\frac{\partial M_{r}}{\partial r}+\frac{M_{r}}{r}\right)=0 \\
\frac{1}{r}\left(\frac{\partial}{\partial r}\left(r \frac{\partial \phi_{0}}{\partial r}\right)\right)=0
\end{gathered}
$$

Equation (17) implies that

$$
H_{z}=\text { Constant }
$$

Since pressure and density are uniform initially, therefore it is clear from (12) that the gravitational potential is balanced by centrifugal force.

Let us allow the small perturbation in the initial state described by (11) as following;

$$
\begin{gathered}
\vec{u}^{\prime}=\left(u_{r}^{\prime}, r \omega+u_{\theta}^{\prime}, u_{z}^{\prime}\right) ; \quad \vec{H}^{\prime}=\left(h_{r}^{\prime}, h_{\theta}^{\prime}, H_{z}+h_{z}^{\prime}\right) ; \\
p^{\prime}=p_{0}+\delta p \quad \vec{M}=\left(M_{r}+m_{r}^{\prime}, m_{\theta}^{\prime}, M_{z}+m_{z}^{\prime}\right) ; \\
\phi^{\prime}=\phi_{0}+\delta \phi ; \quad \rho^{\prime}=\rho_{0}+\delta \rho .
\end{gathered}
$$


Using (21) in (10), ignoring the terms of second and higher orders in the perturbations, and using (12)-(20), we have the following linearized perturbed equations:

$$
\begin{gathered}
\rho_{0}\left(\frac{\partial u_{r}}{\partial t}-2 \omega u_{\theta}\right)=\rho_{0} \frac{\partial \delta \phi}{\partial r}-\frac{\partial \delta p}{\partial r}+\mu_{0}\left(M_{r} \frac{\partial h_{r}}{\partial r}\right) \\
\rho_{0}\left(\frac{\partial u_{\theta}}{\partial t}+u_{r} \frac{\partial}{\partial r}(r \omega)+u_{r} \omega\right)=\frac{\mu_{0}}{2}\left(M_{r} \frac{\partial h_{\theta}}{\partial r}-h_{\theta} \frac{\partial M_{r}}{\partial r}\right) \\
\rho_{0} \frac{\partial u_{z}}{\partial t}=\frac{\mu_{0}}{2}\left(h_{r} \frac{\partial M_{z}}{\partial r}+M_{z} \frac{1}{r} \frac{\partial}{\partial r}\left(r h_{r}\right)-H_{z} \frac{1}{r} \frac{\partial}{\partial r}\left(r m_{r}\right)\right) \\
\frac{\partial m_{r}}{\partial t}+u_{r} \frac{\partial M_{r}}{\partial r}+\frac{1}{2}\left(M_{z} \frac{\partial u_{z}}{\partial r}+m_{\theta}\left(\frac{\partial}{\partial r}(r \omega)+\omega\right)\right)+\alpha m_{r} \\
+\beta\left(-M_{z}^{2} h_{r}+H_{z} M_{z} m_{r}+M_{z} M_{r} h_{z}+H_{z} M_{r} m_{z}\right)=0 \\
\frac{\partial m_{\theta}}{\partial t}-\frac{1}{2}\left(m_{r}\left(\frac{\partial}{\partial r}(r \omega)+\omega\right)+M_{r} \frac{1}{r} \frac{\partial}{\partial r}\left(r u_{\theta}\right)\right)+\alpha m_{\theta} \\
+\beta\left(H_{z} M_{z} m_{\theta}-M_{z}^{2} h_{\theta}-M_{r}^{2} h_{\theta}\right)=0 \\
\frac{\partial m_{z}}{\partial t}+u_{r} \frac{\partial M_{z}}{\partial r}-\frac{1}{2} M_{r} \frac{\partial u_{z}}{\partial r}+\alpha m_{z} \\
+\beta\left(M_{r} M_{z} h_{r}-2 M_{r} H_{z} m_{r}-M_{r}^{2} h_{z}\right)=0 \\
\frac{\partial h_{z}}{\partial r}=0 \\
\frac{1}{\partial t} \delta \rho+\rho_{0}\left(\frac{\partial u_{r}}{\partial r}+\frac{u_{r}}{r}\right)=0 \\
\left(\frac{\partial h_{\theta}}{\partial r}+\frac{\partial \delta \phi}{r}\right)=0 \\
\frac{1}{\partial r}\left(r m_{r}\right)=-\frac{1}{r} \frac{\partial}{\partial r}\left(r h_{r}\right)
\end{gathered}
$$

where $\left(u_{r}^{\prime}, u_{\theta}^{\prime}, u_{z}^{\prime}\right),\left(h_{r}^{\prime}, h_{\theta}^{\prime}, h_{z}^{\prime}\right),\left(m_{r}^{\prime}, m_{\theta}^{\prime}, m_{z}^{\prime}\right), \delta p, \delta \rho$, and $\delta \phi$ are the respective perturbations from basic state in velocity, magnetic field, magnetization, pressure, density, and gravitational potential vector. In the above equations, the dashes have been dropped for convenience in writing.

Since we have considered that the fluctuations in pressure and density take place adiabatically, therefore

$$
\delta p=c^{2} \delta \rho .
$$

Using this equation of state (33) for adiabatic medium, (22) reduces to

$$
\rho_{0}\left(\frac{\partial u_{r}}{\partial t}-2 \omega u_{\theta}\right)=\rho_{0} \frac{\partial \delta \phi}{\partial r}-c^{2} \frac{\partial \delta \rho}{\partial r}+\mu_{0}\left(M_{r} \frac{\partial h_{r}}{\partial r}\right) .
$$

\section{Gravitational Instability}

In order to investigate the stability of the forgoing stationary state, we shall consider the dependence of the perturbation on $r$ and $t$ of the form

$$
\psi^{*}(r) \exp (\sigma t)
$$

where $\sigma$ is frequency of the perturbation.

For this type of dependence of perturbation on $r$ and $t$, we have

$$
\frac{\partial}{\partial t} \equiv \sigma, \quad \frac{\partial}{\partial r} f(r)=\frac{d}{d r} f(r) .
$$

Using the above dependence, the perturbations equations (34) and (23)-(32) assume the following forms:

$$
\begin{aligned}
& \sigma u_{r}-2 \omega u_{\theta}-\frac{d \delta \phi}{d r}+\frac{c^{2}}{\rho_{0}} \frac{d \delta \rho}{d r}-\frac{\mu_{0}}{\rho_{0}}\left(M_{r} \frac{d h_{r}}{d r}\right)=0, \\
& \sigma u_{\theta}+u_{r} \frac{d}{d r}(r \omega)+u_{r} \omega-\frac{1}{2} \frac{\mu_{0}}{\rho_{0}}\left(M_{r} \frac{d h_{\theta}}{d r}-h_{\theta} \frac{d M_{r}}{d r}\right)=0,
\end{aligned}
$$

$$
\sigma u_{z}-\frac{1}{2} \frac{\mu_{0}}{\rho_{0}}\left(h_{r} \frac{d M_{z}}{d r}+M_{z} \frac{1}{r} \frac{d}{d r}\left(r h_{r}\right)-H_{z} \frac{1}{r} \frac{d}{d r}\left(r m_{r}\right)\right)=0,
$$

$$
\begin{gathered}
(\sigma+\alpha) m_{r}+u_{r} \frac{d M_{r}}{d r}+\frac{1}{2}\left(M_{z} \frac{d u_{z}}{d r}+m_{\theta}\left(\frac{d}{d r}(r \omega)+\omega\right)\right) \\
+\beta\left(-M_{z}^{2} h_{r}+H_{z} M_{z} m_{r}+M_{z} M_{r} h_{z}+H_{z} M_{r} m_{z}\right)=0
\end{gathered}
$$

$$
\begin{aligned}
(\sigma+\alpha) m_{\theta} & -\frac{1}{2}\left(m_{r}\left(\frac{d}{d r}(r \omega)+\omega\right)+M_{r} \frac{1}{r} \frac{d}{d r}\left(r u_{\theta}\right)\right) \\
& +\beta\left(H_{z} M_{z} m_{\theta}-M_{z}^{2} h_{\theta}-M_{r}^{2} h_{\theta}\right)=0,
\end{aligned}
$$

$$
\begin{gathered}
(\sigma+\alpha) m_{z}+u_{r} \frac{d M_{z}}{d r}-\frac{1}{2} M_{r} \frac{d u_{z}}{d r} \\
+\beta\left(M_{r} M_{z} h_{r}-2 M_{r} H_{z} m_{r}-M_{r}^{2} h_{z}\right)=0 \\
\frac{d h_{z}}{d r}=0 \\
\frac{1}{r} \frac{d}{d r}\left(r h_{\theta}\right)=0 \\
\frac{1}{r} \frac{d}{d r}\left(r m_{r}\right)=-\frac{1}{r} \frac{d}{d r}\left(r h_{r}\right) \\
\sigma \delta \rho+\frac{\rho_{0}}{r} \frac{d}{d r}\left(r u_{r}\right)=0 \\
\frac{1}{r}\left(\frac{d}{d r}\left(r \frac{d \delta \phi}{d r}\right)\right)=-4 \pi G \delta \rho
\end{gathered}
$$

Since, the above equations involve the variable coefficients, we shall therefore investigate the local stability of the 
above system in the neighborhood of $r=r_{0}$. For this, let us assume that the perturbations have a periodic form in the neighborhood of $r=r_{0}$, as

$$
\bar{f} \exp (-i k r),
$$

where $k$ is the wave number. For this type of dependence, we have

$$
\frac{d}{d r} \equiv-i k
$$

Equations (43)-(45) upon using (49) yield

$$
\bar{h}_{z}=0, \quad \bar{h}_{\theta}=0, \quad \bar{h}_{r}=-\bar{m}_{r} .
$$

Now, substituting (49) in (37)-(42), (46), (47) and using (50), we obtain a system of algebraic equations (with coefficients, in the vicinity of $r=r_{0}$ ) for amplitudes, marked with bars, which can be put in the matrix notation as following;

$$
\left[\begin{array}{cccccc}
\frac{1}{\sigma}\left(\sigma^{2}+Q\right) & -2 \omega & 0 & -i k \frac{\mu_{0}}{\rho_{0}} M_{r} & 0 & 0 \\
P & \sigma & 0 & 0 & 0 & 0 \\
0 & 0 & \sigma & \frac{\mu_{0}}{2 \rho_{0}}\left[\frac{d M_{z}}{d r}-i k B_{z}\right] & 0 & 0 \\
\frac{d M_{z}}{d r} & 0 & -\frac{i k M_{z}}{2} & \sigma+N & \frac{P}{2} & \beta H_{z} M_{z} \\
0 & -\frac{i k M_{r}}{2} & 0 & \frac{P}{2} & \sigma+R & 0 \\
\frac{d M_{z}}{d r} & 0 & -\frac{i k M_{r}}{2} & T & 0 & \sigma+\alpha
\end{array}\right]\left[\begin{array}{c}
\bar{u}_{r} \\
\bar{u}_{\theta} \\
\bar{u}_{z} \\
\bar{m}_{r} \\
\bar{m}_{\theta} \\
\bar{m}_{z}
\end{array}\right]=0 .
$$

Here, $B_{z}=\left(M_{z}+H_{z}\right), Q=k^{2} c^{2}-4 \pi G \rho_{0}, N=\alpha+\beta\left(M_{z}^{2}+\right.$ $\left.H_{z} M_{z}\right), R=\left(\alpha+\beta M_{z} H_{z}\right)$, and $T=\left(M_{r} M_{z}-2 M_{r} H_{z}\right)$.

For the nontrivial solution of the system (51), the determinant of the matrix of coefficients should vanish. Thus, on expanding the coefficient matrix and equating the real part equal to zero, we get the following dispersion relation:

$$
\sigma^{6}+B_{1} \sigma^{5}+B_{2} \sigma^{4}+B_{3} \sigma^{3}+B_{4} \sigma^{2}+B_{5} \sigma+B_{6}=0
$$

where,

$$
\begin{aligned}
& B_{1}=(\alpha+N+R) \\
& B_{2}=\left(8 P \omega+4 Q-P^{2}+4 N R+4 \alpha N+4 \alpha R\right. \\
& \left.+k^{2} \frac{\mu_{0}}{\rho_{0}} B_{z} M_{z}-4 \beta T H_{z} M_{r}\right), \\
& B_{3}=\left(\frac{\mu_{0}}{\rho_{0}} 4 N Q+4 Q R+4 \alpha Q-\alpha P^{2}+4 N R+8 \omega N P+8 \omega P R\right. \\
& +8 \alpha \omega P+k^{2} \frac{\mu_{0}}{\rho_{0}} R B_{z} M_{z}+\alpha k^{2} \frac{\mu_{o}}{\rho_{0}} R B_{z} M_{z} \\
& \left.+k^{2} \beta \frac{\mu_{o}}{\rho_{0}} R B_{z} M_{z} M_{r}^{2}-4 \beta R T M_{r} H_{z}\right), \\
& B_{4}=\left(-P^{2} Q-2 \omega P^{3}+4 \rho_{0} N Q R+4 \alpha N Q+4 \alpha \rho_{0} Q R\right. \\
& +8 \omega N P R+8 \alpha \omega N P+8 \alpha \omega P R-4 \beta Q T M_{r} H_{z} \\
& -8 \omega \beta P T M_{r} H_{z}+k^{2} \frac{\mu_{0}}{\rho_{0}} P^{2} M_{r}^{2}+k^{2} \frac{\mu_{0}}{\rho_{0}} Q B_{z} M_{z} \\
& \left.+\alpha k^{2} \frac{\mu_{0}}{\rho_{0}} R B_{z} M_{z}+2 \omega k^{2} \frac{\mu_{0}}{\rho_{0}} P B_{z} M_{z}+\beta k^{2} \frac{\mu_{0}}{\rho_{0}} R H_{z} M_{r}^{2}\right),
\end{aligned}
$$

$$
\begin{aligned}
B_{5}=(- & 2 \alpha \omega P^{3}-\alpha P^{2} Q+4 \alpha R N Q+8 \alpha \omega N P R \\
& -4 \beta Q R T M_{r} H_{z}-8 \omega \beta P R T M_{r} H_{z}+k^{2} \frac{\mu_{0}}{\rho_{0}} Q R B_{z} M_{z} \\
& +\alpha k^{2} \frac{\mu_{0}}{\rho_{0}} Q B_{z} M_{z}+\alpha k^{2} \frac{\mu_{0}}{\rho_{0}} P^{2} M_{r}^{2} \\
& +\beta k^{2} \frac{\mu_{0}}{\rho_{0}} Q B_{z} H_{z} M_{r}^{2}+2 \omega k^{2} \frac{\mu_{0}}{\rho_{0}} P R B_{z} M_{z} \\
& \left.+2 \alpha \omega k^{2} \frac{\mu_{0}}{\rho_{0}} P B_{z} M_{z}-2 \omega \beta k^{2} \frac{\mu_{0}}{\rho_{0}} P B_{z} H_{z} M_{r}^{2}\right) \\
B_{6} & =\frac{\mu_{0} k^{2}}{4 \rho_{0}} R B_{z}\left(\alpha M_{z}+\beta H_{z} M_{r}^{2}\right)(Q+P) .
\end{aligned}
$$

Equation (52) is of sixth degree in $\sigma$ with all the coefficients real and the coefficients of $\sigma^{6}$ and $\sigma^{5}$ are clearly positive while the coefficients of $\sigma^{4}, \sigma^{3}, \sigma^{2}, \sigma$, and the constant term may be negative depending on $B_{2}, B_{3}, B_{4}, B_{5}$, and $B_{6}$. As per the criteria of Guillemin [20] for the signs of the roots, we get that if $B_{6}$ is negative, then the constant term of (52) is negative, which is sufficient to give the required condition of instability. Therefore, we obtain the following condition for the onset of gravitational instability for the present problem:

$$
k^{2} c^{2}+2 \omega \frac{d}{d r}\left(\omega r^{2}\right)<\left(4 \pi G \rho_{0}\right)
$$

which is the same result as obtained by Bel and Schatzman [9]. 


\section{Conclusions}

In the present analysis, we have investigated the effect of ferromagnetization in the presence of nonuniform magnetic field on the gravitational instability of a homogenous, ferromagnetic dust cloud in the presence of nonuniform rotation. The inequality (54) implies that the Bel and Schatzman criterion determines the gravitational instability of this general problem, thus we can conclude that the ferromagnetism in the presence of magnetic field has no effect on the gravitational instability on the self-gravitating cloud. Comparing this result with result obtained by Dhiman and Dadwal [10] for the gravitational instability of a homogeneous gaseous medium in the presence of nonuniform magnetic field for nonferromagnetic case, it appears that the effect of magnetic field on the criterion for instability has been marginalized by the magnetic polarizability of ferrofluid. Further, from inequality (54) for $\omega=0$, we can deduce that gravitational instability is governed by the Jeans criterion, in the absence of nonuniform rotation.

\section{References}

[1] J. H. Jeans, “The stability of spherical nebulae," Philosophical Transactions of the Royal Society, vol. 199, pp. 1-53, 1902.

[2] S. Chandrasekhar, Hydrodynamic and Hydromagnetic Stability, Oxford University Press, 1961.

[3] T. C. Mouschovias, "Magnetic braking, ambipolar diffusion, cloud cores, and star formation-natural length scales and protostellar masses," Astrophysical Journal, vol. 373, pp. 169186, 1991.

[4] L. Mestel and L. Spitzer, "Star formation in magnetic dust clouds," Monthly Notices of the Royal Astronomical Society, vol. 116, no. 5, pp. 503-514, 1956.

[5] Y. Li, M. M. Mac Low, and R. S. Klessen, "Star formation in isolated disk galaxies. I. Models and characteristics of nonlinear gravitational collapse," Astrophysical Journal, vol. 626, no. 2, pp. 823-843, 2005.

[6] C. F. McKee and E. C. Ostriker, "Theory of star formation," Annual Review of Astronomy and Astrophysics, vol. 45, pp. 565687, 2007.

[7] E. A. Spiegel and J. L. Thiffeault, "Continuum equations for stellar dynamics:" in Proceedings of the Chateau de Mons Meeting in Honour of Douglas Gough's 60th Birthday, Cambridge University Press, 2003.

[8] R. B. Larson, "The physics of star formation," Reports on Progress in Physics, vol. 66, no. 10, p. 1651, 2003.

[9] N. Bel and E. Schatzman, "On the gravitational instability of a medium in nonuniform rotation," Reviews of Modern Physics, vol. 30, no. 3, pp. 1015-1016, 1958.

[10] J. S. Dhiman and R. Dadwal, "On the gravitational instability of a medium in non-uniform rotation and magnetic field," Astrophysics and Space Science, vol. 325, no. 2, pp. 195-200, 2010.

[11] J. S. Dhiman and R. Dadwal, "The gravitational instability of a non-uniformly rotating heat conducting medium in the presence of non-uniform magnetic field," Astrophysics and Space Science, vol. 332, no. 2, pp. 373-378, 2011.

[12] L. Spitzer, Physics of Fully Ionized Gases, Inter Science, New York, NY, USA, 1962.

[13] R. E. Rosenwieg, Ferrohydrodynamics, Cambridge University Press, 1985.
[14] R. V. Jones and L. Spitzer, "Magnetic alignment of interstellar grains," Astrophysical Journal, vol. 146, p. 943, 1967.

[15] A. A. Mamun and P. K. Shukla, "Waves and instabilities in dark interstellar molecular clouds containing ferromagnetic dust grains," JETP Letters, vol. 75, no. 5, pp. 213-216, 2002.

[16] M. I. Shliomis, "Effective viscosity of magnetic suspensions," Soviet Journal of Experimental and Theoretical Physics, vol. 34, pp. 1291-1294, 1972.

[17] M. I. Shliomis, "Comment on "magnetoviscosity and relaxation in ferrofluids"," Physical Review E, vol. 64, Article ID 063501, 2001.

[18] P. Penfield and H. A. Haus, Electrodynamics of Moving Media, MIT Press, Cambridge, Mass, USA, 1967.

[19] D. Kumar, P. Sinha, and P. Chandra, "Ferrofluid squeeze film for spherical and conical bearings," International Journal of Engineering Science, vol. 30, no. 5, pp. 645-656, 1992.

[20] E. A. Guillemin, The Mathematics of Circuit Analysis, John Wiley \& Sons, New York, NY, USA, 1950. 

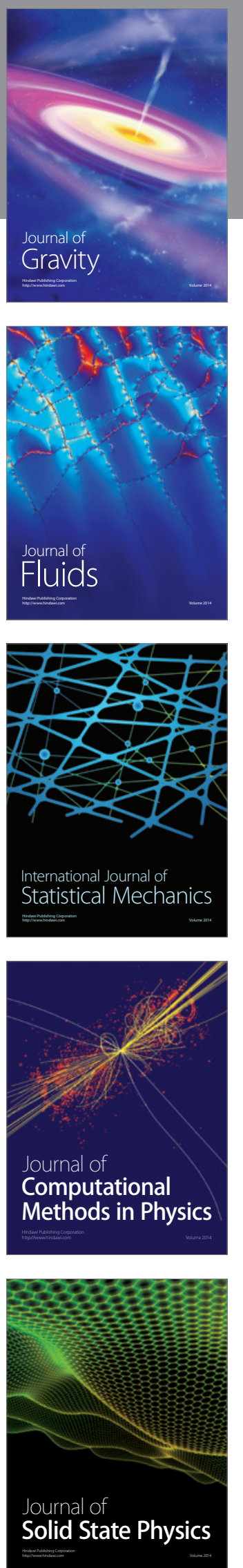

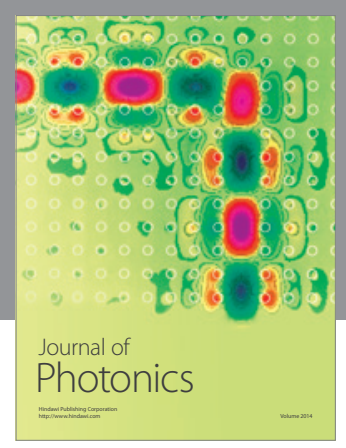

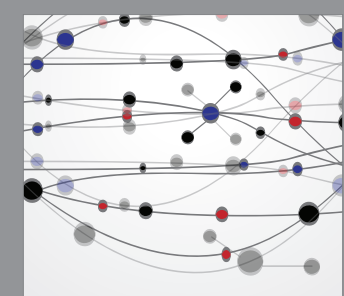

The Scientific World Journal
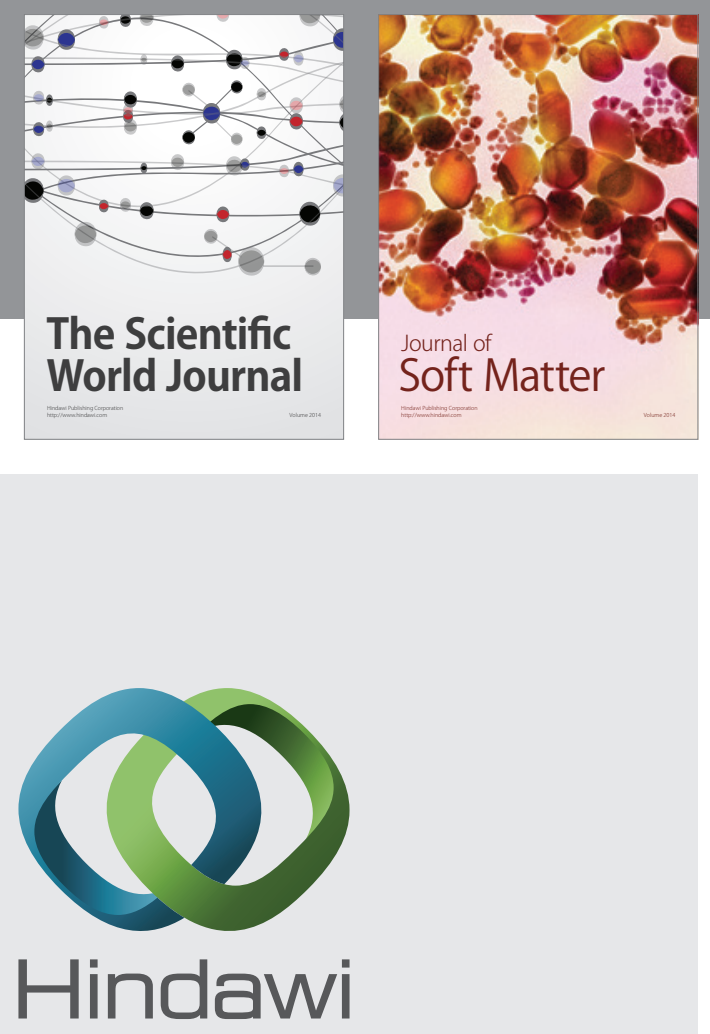

Submit your manuscripts at

http://www.hindawi.com
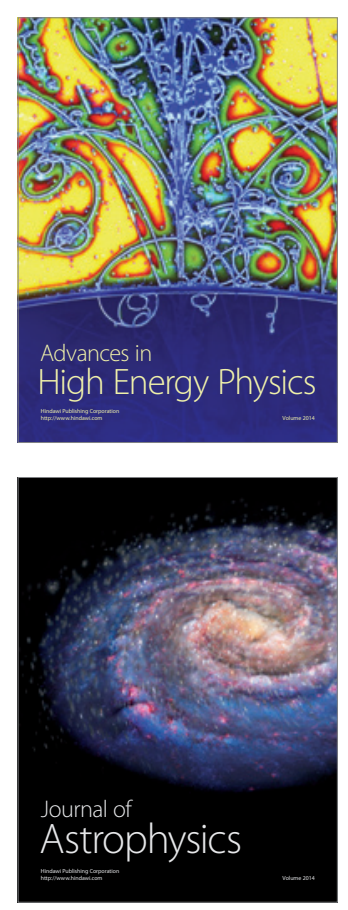
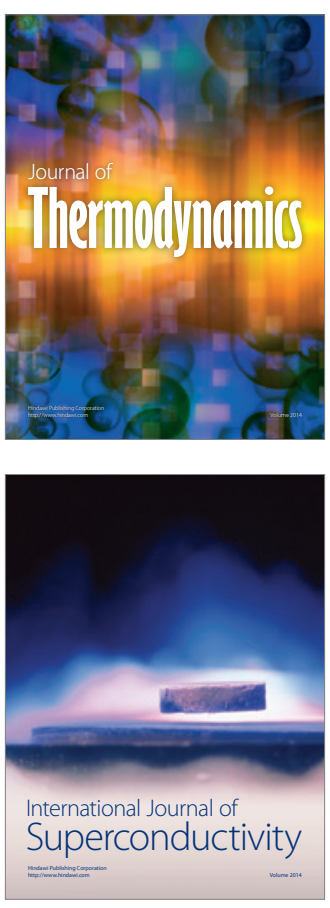
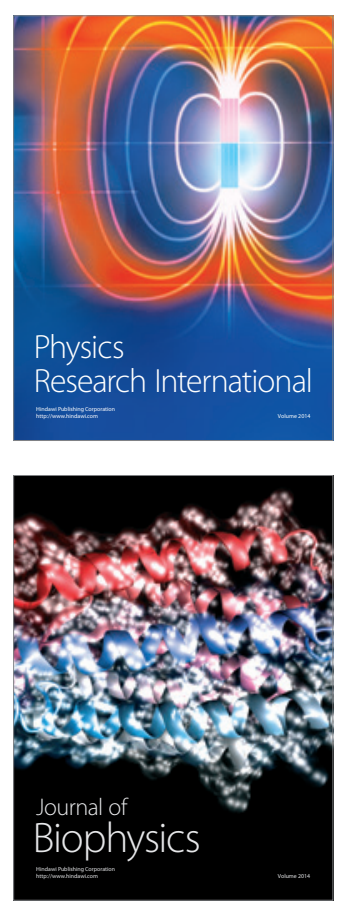
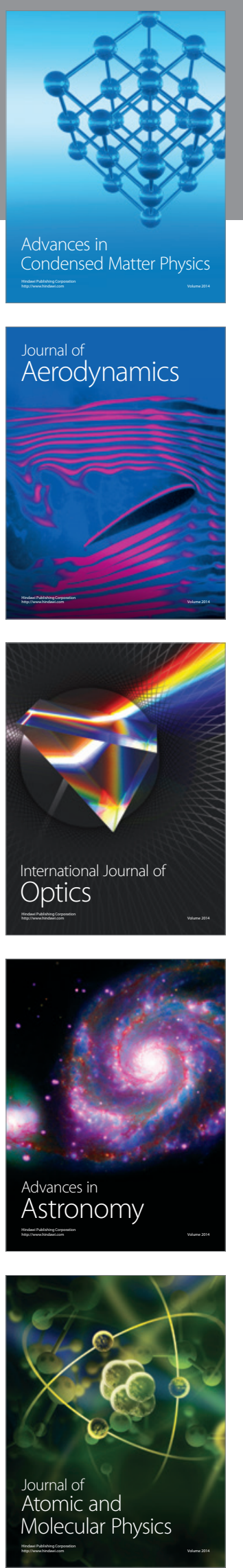